\title{
Analyse Pragmatique Des Modalités Discursives Et Actes De Langage De La Leçon Inaugurale De Kéba Mbaye Du 14 Décembre 2005 À l'Université Cheikh Anta Diop De Dakar
}

\author{
Daouda NGOM*, Mame Sémou NDIAYE
}

Université Cheikh Anta Diop de Dakar, Sénégal

DOI: $\underline{10.36348 / \text { sijll.2020.v03i06.006 }}$

| Received: 06.06.2020 | Accepted: 21.06.2020 | Published: 30.06 .2020

*Corresponding author: Daouda NGOM

\section{Abstract}

This article aims firstly to put up the discursive modalities and speech acts that have a pragmatic value in the text of "The inaugural lesson” by Keba Mbaye. Secondly, it will be a question of analyzing and interpreting the pragmatic value of these speech acts that make successful the communication in terms of persuasion. Thus we will precise the environmental framework of the study. We will inventory and decipher the discursive function of these particular constituent elements of the speech and finally we will put in relation these discursive elements that make persuasive the arguments. The pragmatic approach of communication proposes techniques for the interpretation about what is explicitly or implicitly said and also about the presupposition, will help finding out and analyzing some speech acts from the organizational point of view of the speech. The first part deals with the problematics and offers a presentation of the framework. As for the second part, it focuses on the different discursive mechanisms pointed up by the speaker to accomplish his purpose which is at the same time convincing and persuading the hearers.

Keywords: modality, discourse, speech acts, pragmatics, communication.

Copyright @ 2020: This is an open-access article distributed under the terms of the Creative Commons Attribution license which permits unrestricted use, distribution, and reproduction in any medium for non-commercial use (NonCommercial, or CC-BY-NC) provided the original author and source are credited.

\section{INTRODUCTION}

L'absence d'émotion qui animait Kéba Mbaye dans ses prises de position, l'a hissé au panthéon des figures les plus emblématiques qui se sont succédé à la présidence du Conseil Constitutionnel du Sénégal. Professeur de droit doublé de juge, Kéba Mbaye s'est fait remarquer lors de son passage à la Cour Suprême du Sénégal dont il fut le Président à travers sa fameuse phrase «Les Sénégalais sont fatigués » adressée froidement et vertement à Abdou Diouf, Président de la République, lors de la cérémonie son de installation, le $1^{\text {er }}$ janvier 1981. Cette phrase que le juge Mbaye considère, sous forme de rappel historique lors de son allocution à l'Université Cheikh Anta Diop de Dakar (UCAD), comme «formule lapidaire » (corpus, p 13) [ $\left.{ }^{1}\right]$, reste plus jamais gravée dans la mémoire des Sénégalais, de par le contexte sociopolitique particulier où il a été prononcé. A cela, s'ajoute sa séduisante et vibrante leçon inaugurale sur l'Ethique qui lui a valu un hommage, à titre posthume, qui est la résultante de

\footnotetext{
${ }^{1}$ Ici, corpus renvoie au texte de l'auteur sur lequel nous
} ${ }^{1}$ Ici, corpus
travaillons création de la Fondation Kéba Mbaye. Le sens patriotique qu'on lui accordait se justifiait par son niveau ou train de vie modeste en qualité de Président de plusieurs Institutions du Sénégal. L'homme ne s'est jamais montré, comme des juges d'aujourd'hui l'auraient fait, dans le luxe et commodité que lui offraient ses hautes positions au sein de la république. Sa liberté de ton et son indépendance en tant que juge l'ont hautement inscrit sur le sommet de l'échiquier juridique Sénégalais. Ces faits marquants et ses qualités professionnelles, empreintes de sa carrière de juge, ont façonné une forte admiration chez ses concitoyens Sénégalais, une admiration qui se traduisait par une écoute religieuse des Sénégalais à chaque fois qu'il s'adressait à eux.

La leçon inaugurale de Kéba Mbaye portée sur l'éthique, et qu'il a prononcée à le 14 décembre 2005 à l'Université Cheikh Anta Diop de Dakar, a fait l'objet de plusieurs réflexions scientifiques (panels, colloques, tables rondes, etc.), pour repenser et revivre la problématique de l'Ethique dans des sociétés secouées aujourd'hui par la déperdition des valeurs éthiques et la dégradation des mœurs sociales. 
Le présent article s'inscrit dans une dynamique purement linguistique et essaie de proposer une analyse pragmatique des modalités discursives et des actes de langage relatif à cette communication. Au-delà, il sera aussi question de montrer comment l'auteur a tenté de convaincre ses auditeurs et par quelles stratégies discursives. S'agissant d'un discours social d'invite à une nouvelle orientation de nos comportements socioprofessionnels par rapport à l'éthique, son décryptage sera d'ordre qualitatif. Pour ce faire, nous préciserons l'environnement physique de l'analyse, cela nous permettra de faire un survol de l'ensemble des éléments constitutifs de l'opération oratoire. Nous ferons un inventaire et un décryptage de la fonction performative des actes discursifs de la communication. Et enfin, nous analyserons ces ingrédients discursifs qui participent de la réussite de l'argumentation à des fins persuasives.

\section{Problématique et démarche de l'analyse}

Les nouvelles tendances linguistiques regroupées dans l'appellation englobante de "Sciences du langage» se veulent être un carrefour d'approches connexes allant dans le sens de l'étude scientifique du langage humain, de la langue ou de la parole. Tributaires de la problématique générale du sens, les Sciences du langage proposent d'autres approches pour l'étude de certains aspects du langage humain non pris en compte par la linguistique proprement dite. L'étude du langage humain était considérée comme l'apanage unique de la linguistique ; «La linguistique est l'étude scientifique du langage humain »[1]. Mais cette linguistique ne s'intéresse qu'au mot d'où l'appellation de «la linguistique du mot de Saussure ». La non prise en compte par cette linguistique de certains paramètres langagiers tels que le référant, le sens, le contexte, est à l'origine de la linguistique du discours, de la linguistique du contenu, de la linguistique textuelle, l'analyse du discours, etc., vers la fin des années 60 / 70. Cette apparition de plusieurs linguistiques justifie la naissance de la notion de Sciences du langage. Plusieurs théories émergent de cette situation. Parmi elles, nous avons les théories du langage avec Moeschler et Auchlin [2], la théorie des actes du langage de John L. Austin et John Searle, la théorie de l'agir communicationnel de Jürgen Habermas [3]. Ces théories s'accompagnent de méthodologies appropriées pour les mécanismes d'interprétation du discours.

L'approche pragmatique du discours se base sur la production langagière de l'homme. Cette branche de la linguistique que les uns prennent comme un courant d'analyse du discours à part et les autres comme une conception du langage, a gagné du terrain dans l'analyse du discours de manière particulière, avec une influence forte dans les Sciences humaines et sociale par l'entremise des travaux du philosophe et sémiologue américain Pierce (1839-1914).

C'est pour dire que la fin des années soixante, début des années soixante dix, le discours oral comme écrit a longtemps attiré l'attention des chercheurs. Des chercheurs spécialistes en diverses branches de ces dites Sciences à l'image des analystes de discours, des analystes des interactions, des spécialistes en pragmatique, etc. ont exploré beaucoup de domaines de la communication des individus à travers des approches différentes dont le dénominateur est l'étude du langage. L'un des théoriciens de la philosophie analytique, Searle, affirme que parler « c'est réaliser des actes de langage, des actes comme poser des affirmations, donner des ordres, poser des questions, faire des promesses et ainsi de suite et, dans un domaine plus abstrait, des actes comme référer, prédiquer. » [4].

Pour la conception pragmatique, il y a une connexion entre les actes de langage et l'intention du sujet en communication. De ce fait, les actes de langage englobent presque l'intégralité de l'activité discursive. Ici, la conception pragmatique place la pratique discursive dans le domaine de la subjectivité en prenant en considération toutes les diverses marques de celle-ci. Les influences (ou effets) d'une telle démarche sont remarquables. Le sujet-discoureur s'inscrit dans une logique consistant à faire changer le comportement de son allocutaire, à l'amener à adhérer à ce qu'il dit. Cette opération de modification de comportement ou d'adhésion du sujet qui argumente, s'opère par la variété de ressources octroyées par la langue, partant des «marques explicites de la relation logique (connecteurs) aux marques plus discrètes d'implicitation discursive (présupposition, figures de rhétorique).» (Momar Cissé [5]. Tous ces effets discursifs, à visée persuasive, jalonnent le texte. Le texte de La Leçon inaugurale de Kéba Mbaye est plein de procédés à effets émotifs, affectifs et argumentatifs dont la seule et unique visée est d'amener ses concitoyens Sénégalais à s'approprier l'Ethique, à l'appliquer, à la lettre et sans réserve, dans leurs actions quotidiennes confondues; et partout où ils se trouvent. Reste maintenant la question de savoir comment allons-nous nous prendre pour l'analyse d'un texte argumentatif qui fait appel à la morale, à un changement psychologique et de mode de pratique de l'auditoire? L'approche de la pragmatique communicationnelle mettant en œuvre des techniques d'interprétation du dit et du dit implicite, de la présupposition et du sous-entendu, nous permettra de ressortir et puis d'analyser quelques actes de langage et modalités discursives, du point de l'organisation du discours. Il est important de rappeler que dans un tel exercice discursif, le sujet-discoureur engage l'allocutaire à compléter les éléments restants, autrement dit, les données implicites du discours. L'allocutaire est impliqué, à cet effet, dans un 
processus de «calculs interprétatif » du discours [13, 14].

\section{Mécanismes persuasifs mis au service de son discours}

Le sujet qui argumente capitalise toutes les ressources de la langue fréquemment mis en œuvre de la communication. Il opère un choix de style, de raisonnement et de stratégies qui lui permet de persuader la cible et changer son comportement. Etant par excellence l'outil de communication, la langue s'illustre par la variété de ses ressources «allant des marques explicites de relation logique (connecteurs) aux marques plus discrètes d'implicitation discursives (présupposition, figures de rhétorique). » [5]. Réussir sa communication passe habituellement par la mise en œuvre d'un dispositif discursif capable de toucher ses allocutaires. Le texte de Kéba Mbaye regorge d'actes de langage à effets émotifs, à effets de moralisation, et de prise de conscience qui visent à orienter la conscience des Sénégalais vers l'éthique et il subjugue, par les moyens de la langue, les esprits et les cœurs de son auditoire.

\section{L'opération de contextualisation}

$\mathrm{La}$ prise en considération du contexte d'énonciation dans l'analyse d'une communication constitue un élément d'importance capitale, qui facilite l'interprétation des énoncés. Il s'agit en principe de «tout recours à la situation de communication ». Dans l'allocution de Kéba Mbaye, les déictiques ont une fonction d'appel à l'écoute, à l'attention de l'auditoire. C'est le rôle que Baylon et Mignot leur accordent. Pour ces derniers, les déictiques ont pour fonction «d'attirer l'attention de l'auditeur sur un référent présent dans la situation de parole à l'aide de mot spécifique » [6].

Dans le discours de Kéba Mbaye, l'emploi massif de la deixis temporelle «aujourd'hui », apparue dix fois, du titre à la conclusion du texte, sauf erreur de notre part, renvoie à une pluralité de fonction dans sa communication. L'apparition de la deixis dans l'intitulé de la leçon «L'éthique, aujourd'hui» constitue un procédé de contextualisation du discours. L'auteur attire, d'emblée, à travers ce titrage, l'attention de l'auditoire au contexte dans lequel il place son thème.

Ce cadrage, par le biais de la deixis «aujourd'hui », non seulement présuppose une variété d'entendements, et de perceptions de la notion d'éthique mais également il permet à l'auteur de cerner la problématique de l'éthique dans un contexte précis : aujourd'hui. Le titrage indique explicitement l'angle sous lequel Kéba Mbaye va aborder le sujet qu'il a choisi et le contenu sémantico-sociologique qu'il donnera à l'éthique : «significations en rapport avec le présent ». Il s'agit clairement de la perception des Sénégalais de l'éthique de nos jours, et l'intitulé $\mathrm{du}$ sous-titre «La société face à l'éthique, aujourd'hui» le démontre à suffisance. Du point de vue argumentatif, la deixis «aujourd'hui » constitue une forme d'insistance du sujet-discoureur par rapport à l'importance qu'il accorde au contexte de son discours. Ces formes d'insistance sont, à leur tour, des stratégies discursives servant non seulement à renforcer les idées exprimées mais aussi à les mettre dans un contexte déterminé afin de mieux appréhender leur sens et / ou leurs significations: «De nos jours l'éthique est toujours présente avec un nouveau contenu. » (p 4); « la société face à l'éthique, aujourd'hui » (idem). A travers l'emploi de la deixis « aujourd'hui », Kéba Mbaye appelle à une nouvelle orientation de la conception de l'éthique, partant d'une réflexion générale approfondie et permanente pour la construction d'une société basée sur le principe de l'éthique.

«Il faut aujourd'hui, qu'à travers le diagramme de l'éthique, soit aussi examinés avec soin, non seulement les sciences et les technologies, mais également les pouvoirs étatiques : exécutif, législatif et judiciaire, l'éducation, la conduite de l'étudiant, le rôle de l'enseignant, la fonction d'administrateur, les activités économiques des secteurs primaire, secondaire et tertiaire, la politique en général, le combat pour le pouvoir, la gouvernance, les rapports entre les différents membres de la scène politique, les relations entre gouvernants et gouvernés, le comportement de ces derniers, la communication, la famille, le voisinage, le sport, la culture, les relations internationales, les rapports entre pays riches et pays pauvres et d'une façon générale, comme je le disais il y a quelques instants, l'ensemble des activités et du comportement des hommes pris individuellement ou collectivement, mais aussi des Etats, c'est à dire de leurs représentants. » (p 5)

Le déictique temporel contenu dans ce passage, situe les recommandations du sujetcommuniquant dans une période déterminée: le présent. Le recours récurrent à ce déictique temporel dans son discours, sous-entend des conceptions et / ou perceptions que d'autres avaient de l'éthique dans le passé; et qui sont bien différentes de celles des générations actuelles sur l'éthique. Ces différences de conceptions et de perceptions que nous avons, selon les périodes, de l'éthique apparaissent implicitement dans l'intitulé du sous-titre III «La conception actuelle de l'éthique». L'adjectif qualificatif «actuelle » joue une fonction de référence dans le temps et présuppose, dans la foulée, l'existence de conception « ancienne ou révolue » de l'éthique.

Cette référence dans le temps, par le même déictique, se remarque dans son discours lorsqu'il est question de définir l'éthique : «L'éthique, aujourd'hui, nous apparaît comme un ensemble de règles écrites ou non écrites, inspirées par la morale 
pratique et qui doivent être respectées dans la vie professionnelle et dans la vie de tous les jours. » (p 9)

Même quand il se répète en citant un de ses discours dans le passé, Kéba Mbaye, à travers l'utilisation de la deixis « aujourd'hui », campe à nouveau son discours dans le présent: «Si cette citation est longue, c'est parce que je considère que les mots qu'elle contient ont encore aujourd'hui toutes les raisons d'être redits. Surtout que nous allons vers des compétitions électorales. » (p 14)

D'autres deixis comme les pronoms personnels je, nous et vous participent, d'une manière ou d'une autre, au cadrage de la communication, avec des fonctions discursives bien différentes, du point de argumentatif. Ces déictiques, selon Benveniste [7] caractérisent les instances de discours, autrement dit, les actes par lesquels la langue est actualisée en parole par le locuteur alors qu'il en est disjoint. Dans une communication «à sens unique » (ici la prise de parole par l'auditoire n'est pas envisagée, ce dernier ne réagit que via les applaudissements et acquiescements gestuels), la deixis «je» est une identification individuelle du sujet-discoureur. Ici, son emploi indique une prise de position ou un choix personnel de Kéba Mbaye: «le sujet que $\boldsymbol{j}$ 'ai choisi ... ; « «mais ne vous attendez pas que $j e$ suive à la lettre... (p 3); « Si cette citation est longue, c'est parce que je considère que...(p 14). Par l'emploi du « nous ", l'auteur se meut dans l'auditoire et par là, affiche son implication dans le cadre collectif de prise de position ou bien de tout ce qui concerne la collectivité (nation) par rapport à son invite à l'éthique: «Et comme il n'est pas en notre pouvoir de changer le monde, commençons, nous autres Sénégalais, modestement, mais avec détermination par notre pays. » (5); «Le bien. Nous y reviendrons » (p 6) ; «Il nous ont indiqué ce que doit être et nous savons très bien ce qui est éthique aujourd'hui. Mais nous nous en éloignons. » (11)

Et le «vous » employé renvoie automatiquement à l'auditoire.

Le sujet-discoureur se détache de l'auditoire. Cette démarcation par le biais de «vous », appelle l'auditoire à prendre ses responsabilités, à s'impliquer ou à prendre position par rapport à ce que le sujetargumentant préconise ici comme réflexion: «Je vous invite plutôt....»; «Et d'outre-tombe, je leur répondrai, selon le choix qu'ils auront eu à faire, suivant le cas, heureux : "c'est bien", ou, triste, mais tout de même rancunier : "après tout, vous l'avez voulu. » (p 18).

Dans le discours de Kéba Mbaye, ces différentes deixis sont des actes de langage qui accomplissent la fonction de cadrage de la communication et par la même occasion, mais cette fois du point de vue pragmatique, justifient les conditions de production de son discours.

\section{Mise en ouvre de l'implicitation dans le discours}

Si l'on considère le discours comme un acte ou un ensemble d'actes de langage, qu'il soit écrit ou oral, visant à modifier un comportement, un état d'âme ou simplement un état de chose existant chez le destinataire, on peut concevoir qu'il existe une pluralité d'énoncés langagiers avec des visées pragmatiques différentes. La langue étant un moyen de transmission de messages, de connaissances, d'information, etc. on saurait limiter son utilisation à la production d'assertions, dont le sens se réduirait à la représentation d'un état de chose. C'est dans cette foulée que la philosophie analytique anglaise [8] a prouvé que la langue est avant tout un outil pour agir sur autrui. Cette conception n'est qu'une mise en valeur de la force intrinsèque de tout acte de communication, autrement dit, tout sujetcommunicant, lorsqu'il est en activité discursive dans une situation de communication déterminée, accomplit un acte de langage qui peut comporter plusieurs fonctions discursives. Partant de ces considérations, nous décelons une variété d'actes de langage à visée purement communicative dans l'allocution de Kéba Mbaye. Des emprunts comme celui en Wolof (langue nationale majoritairement parlée au Sénégal) «giiro» sont réalisés ici comme des actes d'amplification de la cupidité et de la roublardise érigées honteusement en règle au sein de la société Sénégalaise :

« Cette assurance vous le devinez, consiste à se prémunir contre des jours futurs durant les quels on est écarté de sa «place » ou de tout. Elle conduit, quand on est encore dans une bonne « place », à rien d'autre qu'à la recherche de biens par tous les moyens, d'où la déviance vers l'enrichissement illicite, le " giiros », la corruption sous toutes ses formes, l'absence de l'amour de la nation. » (corpus, 13)

L'emploi de ce terme wolof, dans ce passage, illustre combien l'auteur est navré par l'attitude non patriotique et non républicaine de certains détenteurs de pouvoir dans son pays. Selon la définition dictionnairique de Jean Léopold Diouf [9], «Giiroo » veut dire en Wolof, «se disputer quelque chose, chacun voulant en tirer la plus grande part ». De ce fait, son emploi dans ce texte traduit une forme de dénonciation acerbe contre l'accaparement illégal des biens de la république, la quête de fortune, la course vers l'enrichissement illicite et de tous les maux dont les détenteurs de pouvoir sont à l'origine. Dans la perception sociologique et linguistique wolof, l'item «giiroo » traduit l'absence de civilité dans la manière de partager ou d'effectuer un tel acte, quel que soit la nature. 
L'emploi de cet emprunt de luxe wolof, avec toute sa charge sémantique plus ou moins péjoratif ici, sous-entend les vols de biens appartenant à l'Etat, les détournements de fonds publics devenus monnaie courante ou érigés en règle dans la gestion des affaires de l'Etat. L'auteur fait usage de ce vocable wolof pour qualifier l'absence d'éthique qui caractérise les pratiques de ceux à qui le peuple a confié temporairement la gestion des affaires de l'Etat. Ici, le recours à cet emprunt permet de décrire, de façon implicite, la nature de notre société d'aujourd'hui et l'auteur l'a précédemment dit, à travers le passage que nous reprenons: «Mais nous nous en éloignons, comme s'il s'agissait d'un principe qui n'a plus cours, dans une société éminemment matérialiste, dominée par le seul souci de l'intérêt personnel, de l'enrichissement et de l'accumulation.» (corpus, p 11)

C'est pour dire que le choix porté sur ce terme est bien muri, pour un auditoire composé majoritairement de personnes qui parlent et comprennent le Wolof bien que nous soyons ici dans un temple de savoir: l'université, où la langue de l'administration, de l'enseignement demeure le français.

D'autres emprunts comme l'expression proverbiale «ad vitam eternam» sont des actes d'insistance qui laissent entendre l'urgence et l'obligation de tendre vers un changement de comportement sans délai. Le passage dans lequel, l'expression figure en fait le point:

«Ainsi, quand j'entends certains parler de l'alternance que j'avais préconisée dix neuf ans avant sa survenance, j'ai le sentiment que pour eux elle est une fin, alors qu'elle doit être répétée ad vitam eternam, pour témoigner de l'ancrage de la démocratie dans nos mentalités et nos mœurs. » (corpus, 14).

Le recours discursif à cette expression proverbiale (latine ou grecque) renvoie à une invite à l'endroit de ses compatriotes à s'inscrire, pour l'éternité, dans une logique de culture, de respect... de démocratie que ces derniers doivent prendre comme l'épine dorsale de notre savoir-vivre, savoirfaire et savoir-être. Dans ses stratégies de dénonciation des comportements malsains de certains gouvernants, Kéba Mbaye fait dans une gymnastique argumentative rude. D'actes de moralisation à d'actes de mépris en passant par des actes de minimisation, d'actes de mise en garde, de taquinerie « forcée », de grondement, de réprimande..., tout y est dans son exercice communicatif. Et tout cela passe sous forme de stratégies discursives.

\section{Stratégies de moralisation: une force occulte de persuasion}

La leçon inaugurale de Kéba Mbaye est sans doute célèbre et écoutée de par son cachet « moralisateur ». L'auteur s'érige, par moments, en donneur de leçons de bonne gouvernance, de patriotisme, de bonne conscience professionnelle, par le biais de l'éthique. Situé entre la morale professionnelle et l'éthique, deux notions étroitement liées et complexes, le discours de Kéba Mbaye fonctionne avec une dose de définitions et de perceptions de l'éthique chez les uns, les autres, d'où la force de frappe affective de sa leçon de «morale ». Ses recours à l'interrogation sur cet état fait, dans le passage ci-dessous, en est une parfaite illustration:

«Debout pour le travail et pour l'éthique avant qu'il ne soit trop tard. Debout en nous rappelant devant chaque choix, chaque action, la pensée d'Emmanuel Kant. Demandons-nous chaque fois que nous sommes tentés d'avoir un comportement non éthique, ce que serait la vie si chacun faisait comme nous. Demandons-nous ce que serait une société de délateurs, de profiteurs, de voleurs, de corrupteurs et de corrompus, d'indisciplinés, d'insouciants, d'égoïstes, de fraudeurs ; la liste est longue mais la réponse est une : ce serait une société vouée à l'échec et peut-être à la déchéance et à la misère matérielle et intellectuelle. Alors, évitons de tels comportements. » (corpus, p 15).

A travers ces actes énonciatifs, l'auteur commence par employer un énoncé par lequel il invite, de façon implicite, ses compatriotes à prendre conscience de l'importance du travail et de l'éthique pour le développement. Le rappel à la morale de Kant qui doit accompagner et guider nos actions et pensées, constitue une stratégie communicative par laquelle l'auteur appelle le sens de responsabilité de chacun dans ses faits et gestes quotidiens ou non. Cette démarche discursive d'appel de prise de conscience socioprofessionnelle ou non, s'assimile à une moralisation de l'auditoire par rapport au travail et à l'éthique. Dans ses mouvements argumentatifs à visée persuasive, l'auteur essaie de faire la morale à son auditoire par un rappel de l'utilité du pouvoir et de l'argent: "Le pouvoir est fait pour servir. Il est passager; l'argent ne sert qu'à satisfaire les besoins; au delà, il est inutile. »(corpus, p 7).

Son exercice de moralisation passe également par une invite aux valeurs cardinales et religieuses telles que le respect de soi et le respect de l'auteur "Le respect de soi et le respect des autres par contre est un bien éternel et sans limite. Dieu luimême le prend en considération dans le jugement qu'il porte et portera sur chacun de nous. » (idem) 
Faire du respect une chose appréciée par le Créateur de l'univers: Dieu, vise à la sensibilité religieuse du public. Il vise à titiller la foi de l'auditoire afin que ce dernier en fasse sa propre affaire.

\section{Stratégies de dénonciation par minimisation et / ou par dévalorisation}

Dans son allocution, Kéba Mbaye passe par les ressources dont dispose la langue pour passer au peigne fin les maux qui endiguent le développement de son pays. L'auteur, à travers son discours, se veut être l'avocat du peuple en descendant en flamme tous ceux qui font de la politique une profession et qui l'utilisent pour se servir et desservir la nation. Pour dénoncer toute forme de politique politicienne, Kéba Mbaye qualifie cette forme de politique comme la chose plus facile du monde, par ricochet, une «profession » qui s'octroie sans mérite. Le passage qui suit en fait la synthèse:

«Que nos dirigeants politiques m'excusent. Mais ils savent tous comme moi que ce n'est pas la politique politicienne qui développe un pays. J'ai écrit dans un recueil de discours et d'allocutions que je me propose de publier bientôt, que ce genre de politique est le métier le plus facile du monde. Il ne nécessite ni études, ni apprentissage. » (corpus, 16).

A travers ce passage, Kéba Mbaye dénonce la mauvaise foi de ces dirigeants politiques qui font dans la politique politicienne tout en sachant qu'une telle activité ou attitude n'a aucun apport pour l'épanouissement de notre pays. L'énoncé «Mais ils savent tous comme moi que ce n'est pas la politique politicienne qui développe un pays» est une accusation contre ces dirigeants qui endorment la population parce qu'ils savent en âme et conscience que la politique politicienne n'est qu'un moyen de se servir et de desservir la population. Dans la foulée, l'auteur peint cette activité comme une activité qui ne mérite aucune considération "J'ai écrit dans un recueil de discours (...), que ce genre de politique est le métier le plus facile du monde. Il ne nécessite ni études, ni apprentissage. »

Cette forme de dévalorisation et de minimisation de la politique politicienne, constitue un appel à de nouvelles orientations des Sénégalais vers des priorités autres que la politique. Le sujetargumentant tente de changer la mentalité des Sénégalais et de l'inscrire sur le chemin qui mène à la recherche de ressources humaines compétentes, dotées d'expertise dans plusieurs domaines comme les sciences et : "Plutôt que d'avoir de grands politiciens, cherchons à avoir de grands médecins, de grands ingénieurs, de grands professeurs, de grands spécialistes de l'économie et des finances et même des savants. » (p 17)
Sa démarche consistant à minimiser la politique dans le cadre du développement, classe la politique au bas du rang des activités qui développent un pays : «Les pays les plus puissants du monde ne sont pas ceux où la politique est reine. C'est plutôt le contraire. (p 17)

Invitant ses compatriotes à revoir leurs comportements et la place « reine » qu'ils accordent à la politique, Kéba Mbaye loue les valeurs des Marocains et les le encense et invite les Sénégalais à s'inspirer d'eux :

«Nos amis les Marocains qui avancent à grands pas vers le développement ont créé une Instance: «Ethique et réconciliation ». Quand on les interroge sur la situation de leur pays, ils parlent non de politique mais plutôt d'éducation, de santé, de salubrité, d'ordre et de discipline. L'essentiel de leur discours est « éthique et développement. »(p 16)

Ce passage panégyrique sanctionnant positivement les efforts louables et salutaires des Marocains, conclu par la bonne note «L'essentiel de leur discours est «éthique et développement» montre, à suffisance, la place de l'éthique et développement dans leurs préoccupations principales.

Cette stratégie de Kéba Mbaye consistant à se transformer en laudateur des Marocain est une autre façon insinuée de dénoncer l'ancrage des Sénégalais dans la politique au point d'en faire une profession. Cette dénonciation s'opère par une stratégie de moquerie et de raillerie sous forme de comparaison à travers le passage ci-dessous: «Quand on interroge un Sénégalais sur la situation de son pays, il parle de remaniement ministériel, de querelles ou de limogeages politiques. »

Cette technique communicative, sous mode de taquinerie et de comparaison, non seulement cherche à inciter les Sénégalais à copier les attitudes patriotiques et les valeurs éthiques des Marocains mais également elle affiche clairement la centralité obsessive et l'attitude maladive des Sénégalais à ne parle quotidiennement que de la politique.

\section{Techniques de mise en garde}

L'opération de mise en garde s'articule sur la problématique du choix que les Sénégalais vont opérer. Les invitant à ne pas négliger les conséquences qui découleront de leur choix éthique ou non, Kéba Mbaye prévient les Sénégalais sur sa probable disparition de ce monde sous forme d'adieux séduisants et émotionnels «Je ne serai peut-être plus de ce monde ». Ces adieux aussi touchants qu'ils sont, traduisent les sentiments, états d'âme et les ressentis émotionnels confondus (souhaits et peur) du Sujetdiscoureur par rapport au choix à faire. Il appelle les Sénégalais à peser le pour et le contre de leur choix: 
«Je ne serai peut-être plus de ce monde quand surviendront les conséquences de ce choix, pour lequel les Sénégalais auront opté en faisant soit ce qui est éthique soit ce qui ne l'est pas. Ce jour là, je suis sûr qu'ils diront en eux mêmes: « et pourtant le père Kéba, avant de partir, nous avait avertis ». (p 18)

A travers ce passage, Kéba Mbaye présuppose des risques et potentiels dangers liés à tout autre choix que celui de l'éthique. S'érigeant en « père responsable » ayant invité ses enfants à faire de l'éthique leur choix, Kéba dégage toutes ses responsabilités par rapport aux conséquences qui adviendront d'un choix non éthique en ces termes « « et pourtant le père Kéba, avant de partir, nous avait avertis »

Se voyant déjà mort «Et d'outre-tombe, je leur répondrai....), Kéba se dit prêt à leur répondre dans sa tombe. La force perlocutive de cet énoncé vise à susciter la peur, l'angoisse chez l'auditoire afin qu'il fasse le bon choix: "selon le choix qu'ils auront eu à faire, suivant le cas, heureux: "c'est bien", ou, triste, mais tout de même rancunier: "après tout, vous l'avez voulu. »

Une telle réponse envisagée met à nu l'insouciance de ceux qu'il considère comme fils, elle leur impute la responsabilité de tout ce qui proviendra de leur choix "après tout, vous l'avez voulu.». L'essence de ses mises en garde renvoie au choix de l'éthique comme principe de vie et de travail et c'est ce que Kéba Mbaye a préconisé précédemment tout en suggérant un élan de patriotisme pour bâtir un Sénégal sur la base de l'éthique: «Alors, debout, comme dit notre hymne, pour bâtir un Sénégal uni autour du travail, de l'éthique et de l'intérêt général. C'est un choix fondamental. On ne paraît plus ringard en le préconisant. »

\section{L'emploi de l'injonction «souple» ou de la suggestion: expression d'une volonté collective}

L'injonction peut traduire, sous un régime plus souple, une directive, une consigne, une instruction. Sa forme la plus habituelle s'exprime, par excellence par le mode impératif, qui ne connait de façon naturelle que la deuxième personne, celle $\mathrm{du}$ destinataire. Nous appelons ici injonction «souple» toute forme d'instruction et appel à un changement de conscience, par le biais du syntagme verbal « il faut $q u e . .$.$) tel que noté dans ce passage ci-dessous:$

«Il faut que le dire et le faire n'obéissent plus à cette nouvelle loi selon laquelle peu importe qu'un but ait été atteint par des moyens illégaux ou prohibés par la morale ou l'éthique, la fin étant finalement le seul justificatif de la parole ou du comportement. » (corpus, p 6), ou encore «Il faut que triomphe l'éthique au Sénégal. » (corpus, p 17)
Ici, l'attitude énonciative du communicant cherche à obtenir de l'auditoire (les Sénégalais) ce qu'il désire: le changement de comportements. L'emploi du syntagme verbal «il faut que » traduit une dose d'insistance de la part du sujet-discoureur sur la nécessité et l'urgence des Sénégalais d'emprunter le chemin de l'éthique, qui doit impérativement se matérialiser dans leurs gestes et paroles de tous les jours. Nous admettons qu'on parle plus généralement d'injonctif quand il s'effectue par le mode impératif, c'est sous cet angle que l'on parle de personne de l'impératif. Ici, nous l'employons suivant la définition que Tomassone lui donne [11] «le locuteur attend du destinataire un acte et non une réplique: les seules répliques sont l'acquiescement, le refus ou le mépris» (...) «le locuteur veut agir sur l'interlocuteur pour obtenir de lui un certain comportement » (1996: 135, cité par [10]).

Dans ce passage, cité haut à titre illustratif, Kéba Mbaye attend des Sénégalais des changements de paradigme comportemental, et rien de plus. C'est ce que nous appelons ici par injonction «souple ». L'auteur ne se contente pas seulement de faire dans l'injonction «souple» en mettant, au devant de la scène, ses concitoyens, il affirme son engagement et implication dans le projet de société basée sur l'éthique qu'il propose à ses concitoyens. Son implication dans la matérialisation des comportements éthiques se traduit à travers l'emploi d'énoncés à visée suggestives: «Alors, évitons de tels comportements. » (corpus, p 15).

A ces procédés liés à la suggestion, s'ajoutent ceux liés à l'interrogation formulée à la première personne du pluriel:

«Demandons-nous chaque fois que nous sommes tentés d'avoir un comportement non éthique, ce que serait la vie si chacun faisait comme nous. Demandons-nous ce que serait une société de délateurs, de profiteurs, de voleurs, de corrupteurs et de corrompus, d'indisciplinés, d'insouciants, d'égoïstes, de fraudeurs ; la liste est longue mais la réponse est une : ce serait une société vouée à l'échec et peut-être à la déchéance et à la misère matérielle et intellectuelle.» (corpus, $\mathrm{p}$ 15)

Cet appel, à tire collectif, à une remise en question devant les choix que nous opérons dans plusieurs situations de notre vie, s'effectue par le biais d'énoncés interrogatifs qui affichent clairement l'implication de l'auteur dans ce qu'il dénonce. Implication assurée par l'emploi de verbes conjugués à la première personne du pluriel.

Toujours dans son abnégation et son engagement à combattre, au même titre que tous les Sénégalais, les maux qui gangrènent la société Sénégalaise, Kéba Mbaye suggère ses concitoyens à 
démasquer ceux qui s'illustrent dans les pratiques non éthiques par l'emploi de verbes conjugués à la deuxième personne du pluriel qui indique l'implication du sujet-suggérant dans ce qu'il préconise :

«Déterminons-nous à débusquer et à écarter sans complaisance, au besoin à punir sans faiblesse ceux qui ruinent ce pays ou le desservent: les corrompus et les antinationaux, les roublards et les paresseux. Cultivons l'honnêteté et le courage au travail. Que chacun, au poste où il est, du ministre au planton, du chef d'entreprise au manœuvre, considère comme reposant sur ses seules épaules, le destin de la nation. Inventons un système qui ne récompensera désormais que la compétence, le travail et la probité ». (corpus, p 14)

Dans ce passage, le sujet-suggérant fait appel à une détermination collective rendue de façon naturelle par le verbe «déterminer » à la deuxième personne du pluriel. Cet appel à une détermination commune est renforcé par une sorte d'invites, d'abord, à l'honnêteté et au courage au travail par le truchement du verbe conjugué «cultivons », et puis à l'ingéniosité pour une mise en place d'une société répondant aux normes éthiques.

\section{CONCLUSION}

Au terme de cette étude, nous avons constaté que La leçon inaugurale de Kéba Mbaye, prononcée le 14 décembre à l'Université Cheikh Anta Diop de Dakar, est remplie de modalités et actes de langage. Kéba Mbaye a fait recours à des procédés communicatifs liés à l'argumentation pour faire adhérer l'auditoire à sa démarche. De l'assertion à l'emploi de deixis en passant par les stratégies de persuasion, Kéba Mbaye a mis en exergue un dispositif à la fois pragmatique et argumentatif pour justifier et étayer ses prises de position par rapport à la problématique et problématisation de l'éthique au sein de notre société.

Riche en termes d'aspects linguistiques liés à la rhétorique, à l'argumentation, à la pragmatique ou tout simplement à la communication, La leçon inaugurale de Kéba Mbaye est au centre des préoccupations des Sciences du langage, par ricochet aux préoccupations des linguistes et des analystes du discours qui essayeront d'étudier sa structure, son organisation et son fonctionnement. L'importance accordée à cette Leçon inaugurale, vu le contexte socioprofessionnel marqué par la perdition des valeurs éthiques, nous a amenés à faire ce diagnostic sur des aspects liés à la pragmatique.

\section{RÉFÉRENCES}

1. Martinet, A. (1960). Eléments de linguistique générale, Paris : Seuil.

2. Moescheler, J. et Auchlin, A. (2000). Introduction à la linguistique contemporaine. Paris: A. Colin/HER (1e éd. 1997).

3. Habermas, J. (1981/1987). La théorie de l'agir communicationnel, Paris: Fayard.

4. Searle, J. (1969/1972). Les Actes de langage. Paris: Hermann.

5. Cissé, M. (2009). "Mouvements argumentatifs dans les textes politiques. Analyse sémiodiscursive de l'Allocution de Nicolas Sarkozy à l'université Cheikh Anta Diop de Dakar», in Anales de la Faculté des Lettres et Sciences Humaines, $\mathrm{n}^{\circ}$ 29/A: 25-45.

6. Baylon, C. et Mignot, X. (1995). La sémantique du langage, Paris: Nathan.

7. Benveniste, E. (1970). «L'appareil formel de l'énonciation », in Langages, 12-17.

8. Austin, J. L. (1962/1970). Quand dire c'est faire, Paris: Seuil.

9. DIOUF, J. L. (2001). Dictionnaire wolof-français Français-wolof, Tokyo: Japon.

10. Riegel, M. (1996). La grammaire méthodique du français, Paris: PUF.

11. Tomassone, R. (1997). Pour enseigner la grammaire. Edition Delagrave.

12. Maingueneau, D. (1999). Les termes clés de l'analyse du discours, Paris: Seuil.

13. Searle, J. (1982). Sens et expression, Paris: Minuit.

14. Searle, J. (1979). «Le sens littéral », in Langue française, $\mathrm{n}^{\circ}$ 42: 34-47. 